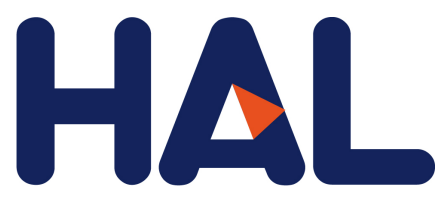

archives-ouvertes

\title{
A Framework for Automated and Composable Testing of Component-based Services
}

\author{
Miguel A. Jiménez, Angela Villotta Gomez, Norha M. Villegas, Gabriel \\ Tamura, Laurence Duchien
}

\section{To cite this version:}

Miguel A. Jiménez, Angela Villotta Gomez, Norha M. Villegas, Gabriel Tamura, Laurence Duchien. A Framework for Automated and Composable Testing of Component-based Services. Maintenance and Evolution of Service-Oriented Systems and Cloud-Based Environments, Sep 2014, Victoria BC, Canada. IEEE Computer Society, 2014. < hal-01055906>

\section{HAL Id: hal-01055906 https://hal.inria.fr/hal-01055906}

Submitted on 14 Aug 2014

HAL is a multi-disciplinary open access archive for the deposit and dissemination of scientific research documents, whether they are published or not. The documents may come from teaching and research institutions in France or abroad, or from public or private research centers.
L'archive ouverte pluridisciplinaire $\mathbf{H A L}$, est destinée au dépôt et à la diffusion de documents scientifiques de niveau recherche, publiés ou non, émanant des établissements d'enseignement et de recherche français ou étrangers, des laboratoires publics ou privés. 


\title{
A Framework for Automated and Composable Testing of Component-based Services
}

\author{
Miguel A. Jiménez*, Ángela Villota Gómez*, Norha M. Villegas*, Gabriel Tamura* and Laurence Duchien ${ }^{\dagger}$ \\ * Department of ICT, Icesi University, Colombia - Email: \{majimenez, apvillota, nvillega, gtamura\}@icesi.edu.co \\ ${ }^{\dagger}$ LIFL - University of Lille 1 CNRS and INRIA Lille, France - Email: laurence.duchien@inria.fr
}

\begin{abstract}
The vision of service-oriented computing has been largely developed on the fundamental principle of building systems by composing and orchestrating services in their flow of control. Therefore, software development is nowadays notably influenced by service-oriented architectures (SOAs), in which the quality of software systems is determined by the quality of the involved services and their actual composition. Despite the efforts on improving their individual quality, adding or replacing services in an evolving system can introduce failures, thus compromising the satisfaction of the system's functional and extra-functional requirements, which is translated as a lack of trust in the SOA vision. Thus, a key issue for the industrial adoption of SOA is providing service providers, integrators, and consumers the means to build confidence that services behave according to the contracted quality conditions. In this paper we present a first version of PASCANI, a framework for specifying and executing automated, composable, and traceable test specifications for service-oriented systems. From a test specification, PASCANI generates a configuration of testing services compliant with the Service Component Architecture (SCA) specification, that can be composed to integrate different testing strategies, being their tests traceable in an automated way. Our evaluation results show the applicability of the framework and a substantial gain in the tester's effort for developing tests.
\end{abstract}

\section{INTRODUCTION}

In the service-oriented computing vision, software development is based on service provisioning, orchestration and composition. This composition of loosely coupled services allows to create flexible, dynamic business processes and applications that pass the boundaries of organizations and involve a wide range of complex computing platforms. The quality of such systems depends on the quality of the involved services and their actual composition and orchestration. However, these systems also evolve, either because some of their services change (e.g., by versions) or because other services with similar, but improved, functionalities appear, or because of new system requirements. Given that services are replaceable by definition, and they usually come from diverse third-party providers, this evolution is performed by adding or replacing them in chains of service compositions. Nonetheless, even though services are usually tested individually in their sources of origin, adding or replacing them can introduce unit, integration, or system level failures, thus compromising the satisfaction of functional and extra-functional requirements (i.e., the system quality).

To maintain service-oriented systems' quality, testers develop, compose, and execute service tests in the required deployment configurations. This process is error prone, and time and effort consuming for several factors: (i) the tester must analyze by hand the services to test, before specifying the actual tests in the service implementation programming languages, being these languages possibly different and not tailored for testing; (ii) the number of service assembly configurations valid to achieve the system goals can be high (e.g., when implementing dynamic binding), and so the respective testing configurations; and (iii) ideally, the testing services should be reusable, composable in larger testing artifacts, and traceable by themselves.

In this paper we present PASCANI, a framework that assists testers in the specification and execution of automated, composable, and traceable tests, which are compliant with the Service Component Architecture (SCA) specification [1], for service-oriented systems. Our framework comprises two subsystems. The first is the PASCANI analyzer, a mechanism for analyzing the possible service configurations to test, which generates a test specification template from a set of selected components and services. The second is the PASCANI language and its implementation, a domain specific language for specifying tests through the definition of test modules. A test module specifies the components and services to test, the test cases for verifying the system's functional and extra-functional requirements, and a test strategy, which specifies how to execute the test cases. For the composability, the language allows to import other test modules and compose them in the test strategy through a set of compositional operators. From a module specification, PASCANI generates a configuration of testing components, and deploys and executes it using FRASCATI [2], an execution middleware for SCA-compliant systems.

To evaluate our approach, we integrate PASCANI into a service-oriented online shop retailer (OSR) application. Our results show the applicability of the framework by generating, deploying and executing configurations of composable tests on the services of the service provider, and the compositions of the service integrator. Our validation demonstrates how PASCANI saves time and effort of developers.

The remainder of this paper is organized as follows. Section II presents the paper motivation by identifying existing challenges for testing service-oriented systems and introducing our case study; Section III presents the main elements of the PASCANI framework; Section IV presents how PASCANI supports test composition; Section V presents the evaluation of our framework; Section VI discusses related work; finally, Section VII concludes the paper.

\section{Motivation}

In this section we analyze selected challenges for testing software services, and introduce our case study. 


\section{A. Revisiting Testing Challenges in Software Services}

Even though it is well known that testing is a limited technique for verifying the correctness of a software system (i.e., testing cannot show the absence of faults, but only their presence), it has demonstrated its practical utility in given scenarios. For example, in industrial settings where an acceptably stable software system evolves after years of use, regression testing is a common alternative to formal verification as a way to maintain achieved levels of quality. In this case, formal verification is not affordable not only because of its cost but also especially because software services are provided by third parties, executed in their infrastructures and made available through the Internet, without access to their source code. Of course, this kind of services, developed and run in extramural conditions (i.e., beyond the walls of the software development organization that depends upon their integration), limits the applicability of traditional white-box testing techniques. Moreover, these services are usually provided without corresponding testing services, not even with instructions for testing them. On the one hand, for service providers test development is costly, not only because its engineering effort but also because the execution of functional and extra-functional tests in their infrastructures can degrade quality attributes of the provided services themselves. On the other hand, for service integrators it is reasonable to require minimum guarantees on the quality of the services to integrate and compose, at both functional and extra-functional levels. Given the benefits and despite its limitations, testing is widely used in practice to provide confidence in software services quality [3], [4], [5].

In the next sections, we present the specific testing challenges addressed in this paper. It is worth noting that, even though we target service testing, our approach follows the vision of the service-oriented architecture (SOA) as based on the service-component architecture (SCA) [6], [1]. That is, we realize services through software components and corresponding service-component middleware, which ensure interoperability with software services implemented in common technologies and exposed through well-known protocols.

1) Composability: The first challenge implied by the SOA/SCA vision is the need for testing components or services that can be composed and reused effectively. In effect, in this vision software development is based on service provisioning, discovery and composition in a worldwide context. Thus, as software systems involves the integration and composition of services from third parties, it should be reasonable to expect that corresponding testing artifacts (i.e., software components) should also be composable in order to maximize their costeffectiveness, leveraging their testing value beyond their original goals. Then, "service-based system tests" for ensuring requirements satisfaction could be seen as a hierarchy of reusable and composable tests, starting from functional ones at the unit (i.e., individual service) level, and building up through layers of integration tests (i.e., for composed services), and finally, a set of extra-functional tests (i.e., at the system level, for instance measuring the performance of the resulting combined execution of own and third-party integrated services).

Furthermore, this challenge is exacerbated by the extramural nature of the services to compose, given the lack of control over the respective testing provisions. Therefore, service integrators require tools that assist them in the specification and implementation of testing artifacts. Naturally, an adequate testing language with precise semantics is also required for expressing not only testing functionalities but also, and more importantly, how to compose these functionalities and artifacts, at different levels of application. For instance, for providers, services are endpoints of software units without specific contexts of use, and thus, they are mainly concerned about unit tests. However, unfortunately they usually provide only PDF instructions to perform manual tests, in the best of the cases. Service integrators, on the other side, usually must develop not only unit tests for these services, but also integration and system tests, in order to have minimum guarantees of having a whole system satisfying its requirements in its specific contexts of operation.

2) Traceability and Logging: A second challenge for testing services is the development of controllable logging mechanisms and standardized trace formats, applicable both to individual tests and to compositions of them, and deployable with testing artifacts automatically.

Service providers and integrators expect to deliver and compose services enabled with control and monitoring functionalities over their properties and behavior. Similarly, in service testing traceability and logging are crucial mechanisms to provide precise information to diagnose and identify the root cause of anomalies. On the one hand, service traceability refers to the capability of tracking the states and attributes of services. From the perspective of service testing, traceability should produce trace information about two aspects: sub-test and service invocations in a test, and respective partial and completed test results according to the test objective. Based on this information, human testers can detect faulty services or services that are contributing the most to satisfy or dissatisfy given requirements, such as quality attributes. On the other hand, logging complements traceability by recording sensitive information about the quality of services, which is expected to be registered consistently from service and test execution. This information should be gathered dynamically from the execution of test cases and corresponding monitor probes in the target system. Traceability in service testing comprises two dimensions: behavior traceability, which refers to the degree a service facilitates the tracking of its internal and external behaviors; and trace controllability, which concerns the customization of tracking and logging functionalities [7]. Moreover, software developers usually focus on tracking the internal behavior of services rather than the external ones. As a result, services are generally better instrumented to support logging in unit tests than in integration and system tests, despite logging external behaviors are more relevant in distributed environments such as in services.

3) Test Specification and Expressiveness: The third challenge is the definition of adequate languages for specifying tests, with appropriate syntax and semantics, power of expressiveness, efficiency, and level of abstraction. Languages with these characteristics would allow testers to construct better tests, easier to understand, reuse, compose, trace, and maintain.

Traditionally, software developers implement test artifacts using ad-hoc approaches that are based on technologydependent test management tools or frameworks [8]. Nevertheless, this way of implementing tests leads to unmanageable sets of testing artifacts written in different general-purpose 
programming languages, that is, not tailored for specifying tests. Moreover, services are usually published through interface definition languages (IDLs) such as WSDL, which testers must analyze for specifying the testing operations, including the translation of methods and parameters between the IDL protocols, and the interactions (composition) with other services, a process prone to errors. Thus, more than implementing tests assisted by technology-dependent tools, SOA/SCA requires domain specific languages (DSLs) that allow software testers to specify testing components in more manageable, standardized ways. The characteristics of such DSLs for specifying tests easier to understand, reuse, compose, trace, and maintain should be [9]:

Expressiveness: DSLs must be expressive enough to capture all the necessary concepts in service testing. That is, it must semantically support the full generation of test concepts and required component configurations with corresponding test cases and strategies, automatically.

Adequate level of abstraction: DSLs are more effective when their notations and abstraction level are closer to the domain experts language. For instance, in general, software developers are more familiar with programming languages than with formal specification languages and verification processes. Nonetheless, neither of these two kinds of languages (programming and formal) are well suited specifically for testing.

\section{B. A Running Example}

The following case study is used along this paper to explain the application of our proposed testing framework. In electronic commerce, an OSR application allows customers/users to shop for products and services through the internet. For this, users browse the catalog of offered products and select the ones to be purchased by adding them to a shopping cart. Furthermore, with the proliferation of personal context and user-centric recommendations, users expect from OSR applications product recommendations based on their preferences and situations. To complete the purchase, users initiate the checkout process by entering the required information about delivery and payment preferences. Delivery preferences include information such as the shipping address and the delivery service selected by the user. Payment information includes data about the preferred payment method such as the credit card number, security code, holder name and billing address. To recommend products relevant to the user, complete the checkout process, and afterwards confirm the delivery of the order, OSR applications compose several services provided by third party applications. In particular, for recommending relevant products, OSRs may implement recommendation systems, for example based on collaborative filtering techniques, that in turn can consume third party services to perform computational expensive operations such as matrix multiplication [10]. Similarly, for completing the checkout process, OSR applications typically use a postal address verifier (PAV) service to verify that the shipping and billing addresses do exist; a credit card number verifier (CCNV) service to confirm all the information about the credit card registered by the user; and a delivery service (DS), with its corresponding delivery tracking service (DTS), to dispatch the order and allow the user to monitor the delivery process. Several instances of these services are already run on the internet and available to be used, both manually by humans and programmatically through wsdl interfaces.

\section{THE PASCANI FRAMEWORK}

SCA defines a programming model to build software systems based on a component model underlying the SOA design principles. SCA provides specifications for the creation of components and their composition to develop complete applications. Components can rely on different environments and technologies, given that SCA is independent of implementation and communication mechanisms that are specified in the implementation and binding models [1].

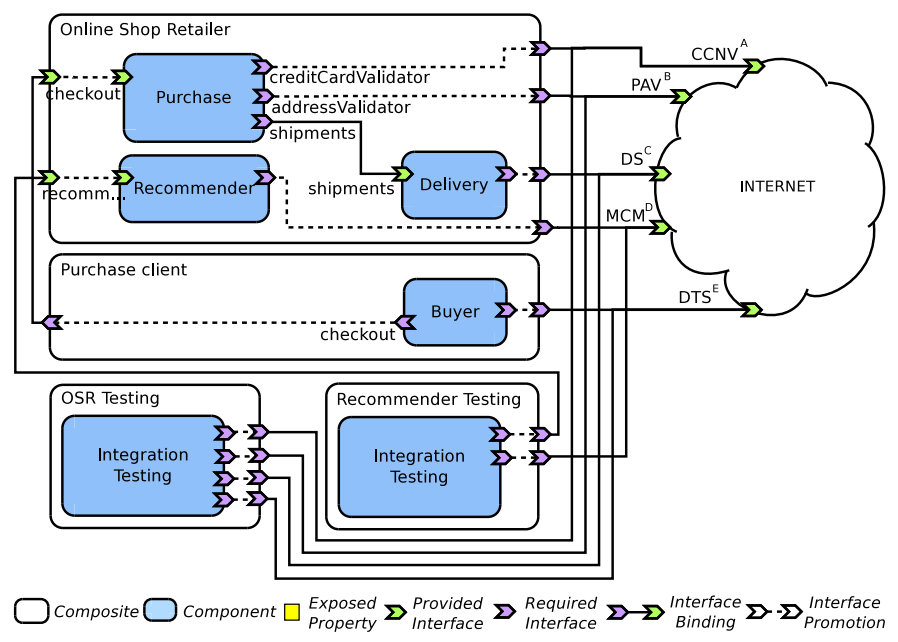

Referenced services are:

(A) https://ws.cdyne.com/creditcardverify/luhnchecker.asmx?wsdl CheckCC(number);

(B) http://pav3.cdyne.com/PavService.svc?wsdl VerifyAddress(address);

(C) http://ws.dev.aramex.net/shippingapi/shipping/service \_1\0.svc?wsdl CreateShipments(request);

(D) host="192.168.160.59" port="1099"

multiply(matrices); and

(E) http://ws.dev.aramex.net/shippingapi/tracking/servicel_1\_0.svc?wsdl TrackShipments(request)

Figure 1: SCA diagram for the Online Shop Retailer example.

Figure 1 depicts a partial view of the SCA architecture, including integration testing components, for the OSR application described in Sect. II-B. This figure will be used in the following sections to explain the PASCANI framework. The legend in the figure presents the main artifacts of SCA.

PASCANI comprises two subsystems: (i) the component and service analyzer, which generates a test specification template from a set of selected components and services; and (ii) the testing specification language, whose design and implementation includes a translation model (i.e., a compiler) and an execution model. That is, the framework assists users (i.e., human testers) in both describing the system to be tested in terms of its components and services; and specifying test modules with the corresponding test cases and strategies for verifying the system's functional and extra-functional requirements. Naturally, the analyzer generates the test specification templates in the syntax defined by the PASCANI language. Finally, the framework deploys and executes the components that realize the test modules.

PASCANI is a valuable testing framework for service providers, integrators and consumers, since it supports them in 
the cost-effective specification and composition of test cases and strategies (i.e., test modules). Furthermore, in the case of service integrators and consumers, PASCANI is particularly useful for composing testing services, exposed by service providers, despite the technology used to develop them. The current version of the PASCANI framework is available from its $\mathrm{SVN}$ repository at http://pascani.org upon request.

This section focuses on explaining the elements of the framework that allow the specification of test modules from the perspective of the service provider. For this, we elaborate on the scenario of a third party service for matrix multiplication, as introduced in our running example (cf. Sect. II-B). In this case, the service provider uses Strassen's algorithm for implementing its matrix chain multiplication $(\mathrm{mcm})$ service (cf. Fig. 1), and uses PASCANI for the specification and composition of its tests.

\section{A. Language Specification}

The main unit for specifying tests in PASCANI is the test module. PASCANI defines two types of test modules: basic and composed. A basic test module does not compose other test modules and is intended only for two testing targets: unit and system testing. In contrast to basic modules, composed test modules reuse other test modules, that may have different testing targets. A test module specifies the system's components and services to be tested, as well as the test cases with their corresponding execution control flow. We also refer to this control flow as the test strategy of the module. Modules are the mechanism to both specify a deployable system and its test components, specify test strategies, and compose tests. For this, a PASCANI module defines two mandatory blocks named system structure and tests, and an optional block named control. Control blocks are similar to main methods in programming languages such as Java and $\mathrm{C}++$, thus they are optional in modules that do not specify a test execution strategy.

Listing 1 illustrates the structure of a basic PASCANI module. This module tests correctness in the implementation of the classical Strassen's algorithm that is used as the basic solution for our case example (cf. Section II-B).

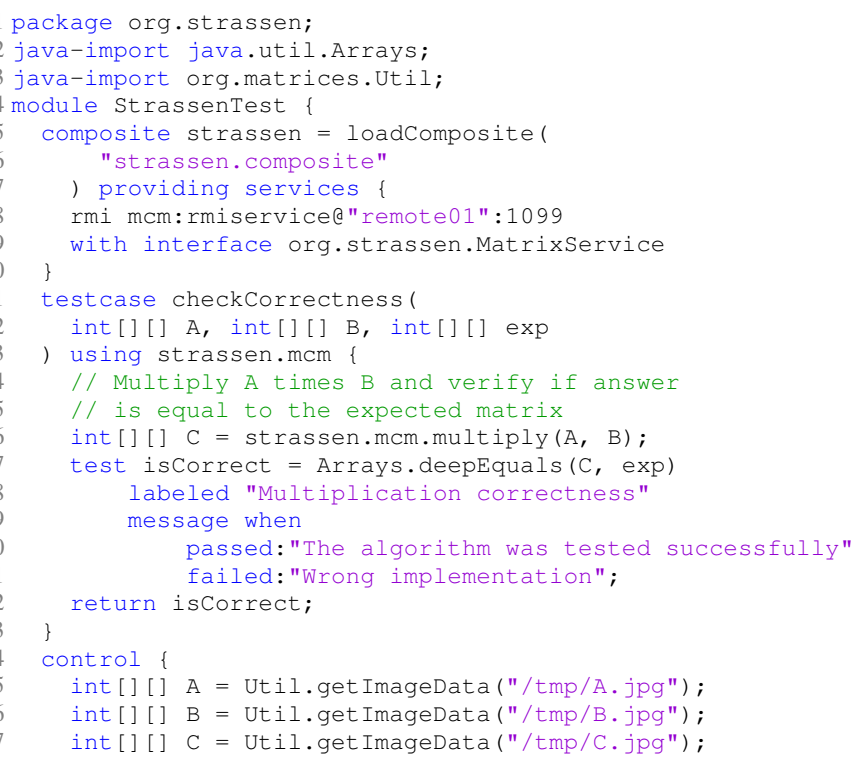

1 illustrates the specification of a system architecture with only a known component named strassen that provides an RMI service named $\mathrm{mcm}$.

Test Block. This mandatory block allows the definition of one or several test cases using the reserved word testcase. A test case specifies a sequence of instructions and use the services defined in the components specified in the system structure block. The final result of executing these instructions evaluates to a test value we call verdict. test is a primitive type introduced by PASCANI. In PASCANI, the verdicts are values in the set $V=$ \{passed, failed, inconclusive, exception $\}$. Lines 11-19 in Listing 1 specify the test case named correctness to verify whether the service strassen.mcm multiplies a pair of matrices as expected. The verdict of the test is returned through the variable iscorrect (cf. line 18).

Control Block. This optional block allows the definition of test strategies, that is, the execution control flow that specifies (i) the test case invocations that will be executed by the module; (ii) their execution order; and (iii) the way of operating or composing their verdicts to obtain the final result. Moreover, control blocks may contain programming-language specific instructions (e.g., Java statements) to prepare the test environment (e.g., to load test data as shown in lines 21-23 of Listing 1), which of course must be executed before calling the test cases.

\section{B. Translation Model}

PASCANI translates test module specifications into SCA component configurations and corresponding programming language implementations (i.e., Java for this version of the framework). Furthermore, PASCANI provides an interface to deploy and execute the generated components using the FRASCATI middleware [2].

From the information specified in the three blocks of a test module, PASCANI generates the corresponding SCA configuration as follows (cf. Fig. 2). The services of components specified in the system structure block are translated into SCA references that are bound to testcase and control components in order to be tested (cf. service $\mathrm{mcm}$ in component Matrix is bound to 
StrassenTest_Group.StrassenTest_checkCorre ctness_strassen_mcm); each of the test cases and the control block are translated into components (cf. StrassenTest_checkCorrectness and StrassenTest_Control) and encapsulated together in the same composite (cf. StrassenTest_Group). Every generated component is equipped with two standard services: run, that is used for starting the execution of the component, and control, for controlling the logging functionalities (e.g., the level of detail of the messages to $\log$ ). In our example, component strassenTest_Control has references to all of the testcase components within StrassenTest_Group, given that it must realize the test strategy as well as the control interface for the logging and traceability of test cases execution. Finally, component Logger records log messages, and component Runner allows the tester user to start the execution of the test module and control the logging level of detail through services Runner.run and Runner. control, respectively.

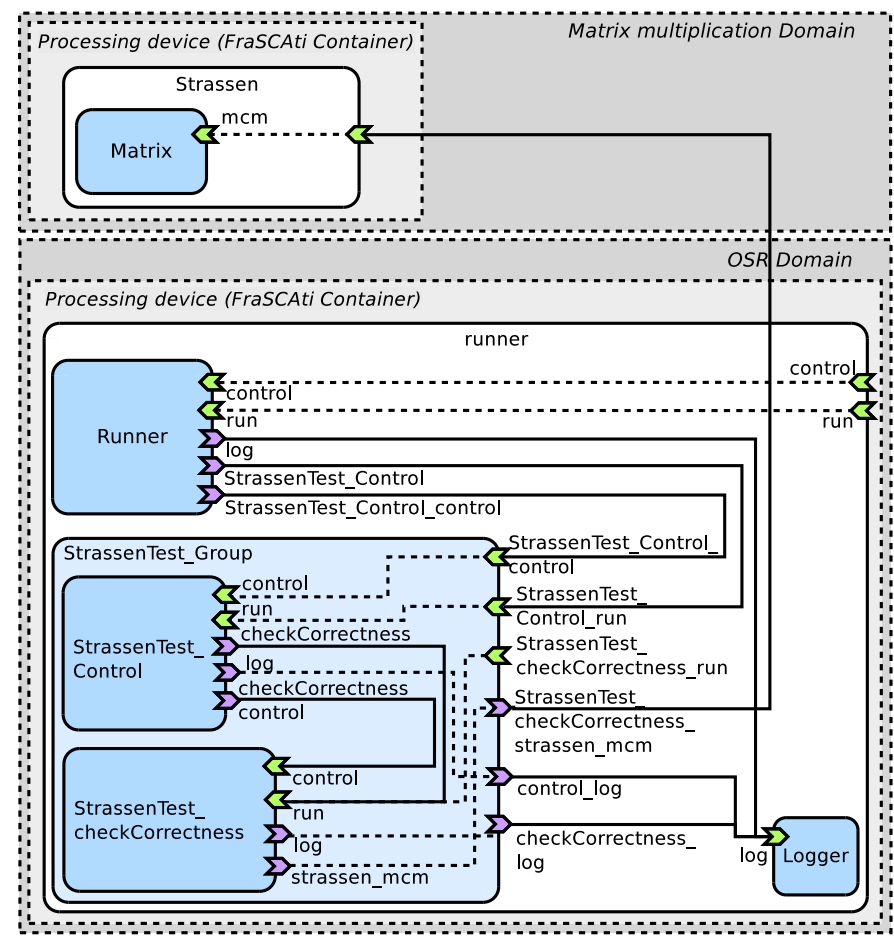

Figure 2: SCA configuration for the StrassenTest module.

\section{Execution Model}

The execution model of PASCANI relies on the standard SCA interfaces, services, and references that are defined for each component in the translation model. The execution model defines the way a test strategy is executed through the behavior of the generated components.

The sequence diagram depicted in Fig. 3 represents the execution model of the StrassenTest module specified in Listing 1. The diagram is adapted to the SCA domain. That is, participants represent the components of the SCA configuration for the StrassenTest module, and messages correspond to the services that allow the communication among them. For simplicity, this sequence diagram focuses on the interactions among the components that realize the test strategy, and omits the ones associated with logging and control mechanisms.

The steps of the execution model for testing the classical implementation of Strassen's algorithm that multiplies two matrices (cf. component Matrix in Fig. 2) is as follows. A Testclient starts the execution of the test module by invoking service Runner. run, which in turn invokes service StrassenTest_Control.run to initiate the execution of the control, that is the test strategy. The control starts the execution of each test case, as specified in the control block of the test module, by calling the corresponding run methods. Afterwards, each test case component invokes the service or services to be tested. In the case of the Strassentest module, the test strategy is composed of only one test case that is implemented in component StrassenTest_checkCorrectness. To perform the actual test, StrassenTest_checkCorrectness consumes service Matrix.mcm, gets the result of the execution, and validates this result with respect to the specification to return the verdict of the test to the control block. Finally, the test ends by generating the test report.

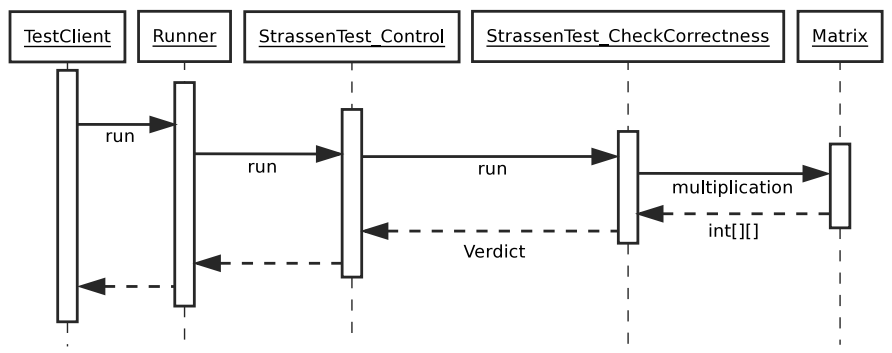

Figure 3: Sequence diagram for the execution model of the StrassenTest module.

The execution model of PASCANI also comprises the monitoring of properties and behaviors of the testing components. PASCANI components expose services to allow the control of their logging mechanism by other SCA components, and through them by users. Furthermore, test components can be monitored by reading the generated log, which corresponds to the traceability of the test strategy execution. By default, PASCANI generates log registries at the most detailed level, that is for the test case execution. However, users can turn off the logging functionalities for all the components in a test module or for selected components of any type (i.e., module, control, and test case components) through the service Runner.control.

As shown in Fig. 2, logging in PASCANI is performed through the component Logger, thus centralizing all log and respective tasks.

\section{Composing Tests with PASCANI}

From the testing perspective, the evaluation of a software application's quality depends not only on the results obtained from the execution of test cases, but also on the quality of the tests themselves [11]. On the one hand, several testing techniques exist for evaluating the fulfillment of functional and extra-functional aspects of quality, such as regression testing and performance testing, respectively. On the other hand, the quality of a set of test modules is usually evaluated 
against selected test criteria, for instance by measuring the coverage (i.e., percentage) of code that is executed when the test modules are applied.

Even though the test module is the basic testing unit of reuse in PASCANI, the key factor for achieving composability and traceability of test specifications resides in its threeblocks structure. More concretely, the reusability of PASCANI testing modules is achieved by three main characteristics: (i) integration of programming language statements within the control block test definition; (ii) modularity of tests; and (iii) composability of deployable testing components and the exposure of services for controlling test traceability and logging.

This section illustrates the principles used in the design of the PASCANI language for defining the composition operators used to realize both testing strategies and tests quality evaluation. These operators compose testing components not only generated by PASCANI, but also other testing components from third parties, as long as they either conform to the SCA specification, or are exposed as testing services. Moreover, to support the composition of tests results, PASCANI defines the operators and, or, xor, and not, associated with its verdict primitive type. Thus, test results in PASCANI are operated similarly as boolean expressions in programming languages, whereas test modules are composed with richer semantics, as follows.

\section{A. Regression Testing}

This testing technique re-tests a system or component to verify that performed modifications in its code did not introduce unintended effects. In other words, after being changed, software artifacts that previously passed known tests should still pass them, except of course when the related requirements also change. Regression testing is applicable at the unit, integration, and system levels to maintain the achieved quality of an existing software system in both functional and extrafunctional aspects.

In PASCANI, previously developed test modules $T M_{1}, \ldots, T M_{N}$ of any kind can be reused and composed for regression testing through the regression test operator, formally denoted as $\uplus$. In the language, the regression composition is expressed as regression $T M_{1}, \ldots, T M_{N}$ \} . The test modules are executed in sequence, avoiding repetitions in them and in service execution.

Definition 1: Given a set of test modules $\left\{T M_{1}, \ldots, T M_{N}\right\}$, the regression test that composes them, denoted as $T M_{1} \uplus \ldots \uplus T M_{N}$ and abbreviated $\biguplus_{i=1}^{N} T M_{i}$, is defined as:

$$
\biguplus_{i=1}^{N} T M_{i}=\bigwedge_{i=1}^{N} T M_{i} \cdot \operatorname{control}()
$$

That is, the result of the regression test composition is the and of the verdicts returned by the test control blocks of the corresponding test modules. Nonetheless, it is worth noting that the semantics of the regression-testing composition operator, in addition to the semantics of the verdict and operator, also implies the automatic redirection of logging messages and traceability information to a unified Logger component in the generated configuration. At runtime, the execution of the test returns the verdict, and as a side-effect, it produces a record of the verdict results of the test modules included in the composition.

\section{B. Performance Testing}

The goal of performance testing is to verify whether the system meets specified performance requirements such as response time, latency, and throughput. This testing technique applies at the unit, integration, and system levels.

Performance tests can be executed either by measuring the execution times of all the components separately and computing the overall performance by adding them, or by measuring the overall execution time at the outer scope of the service invocation. The problem with the latter is that the user may need to have a more detailed analysis of the components individual performance and in this case must include manually the performance "probing" instructions as part of the test strategy specification, thus leaving out of consideration other performance-related aspects such as networked communications time.

In PASCANI, previously developed performance test modules $T M_{1}, \ldots, T M_{N}$ can be composed not only for testing the overall performance of a whole system but also for profiling the services that are contributing the most to performance problems. Both functionalities are realized through the performance-test operator, formally denoted as $\cap$. In the language, the performance composition is expressed as performance $\left\{T M_{1}, \ldots, T M_{N}\right\}$. Although the test modules are executed sequentially, there can be an alternative semantics executing them in concurrent threads. However, as the results are both summed up and also reported by test module, and the concurrent execution depends a lot on the deployment configuration using more or less machines, we found not convincing arguments for implementing this second interpretation.

Definition 2: Given a set of test modules $T S=$ $\left\{T M_{1}, \ldots, T M_{N}\right\}$, the composed performance test of $T S$, denoted as $T M_{1} \cap \ldots \cap T M_{N}$ and abbreviated $\prod_{i=1}^{N} T M_{i}$, is defined as:

$$
\prod_{i=1}^{N} T M_{i}=\sum_{i=1}^{N} \text { Pascani.elapsedTime }\left(T M_{i}\right)
$$

To realize this definition, the PASCANI framework associates a set of attributes to every user-defined test module. These attributes are referred through the meta-variable Pascani (i.e., Pascani.elapsedTime $\left(\mathrm{TM}_{i}\right)$ refers to the elapsed time of the test module $\mathrm{TM}_{i}$ ).

The semantics of the performance-testing composition operator, besides accumulating the elapsed times of the corresponding tested services, activates the recording of their elapsed times of the executed services in the generated configuration at compile time. For having more confident results, each test module is executed a number of times that can be configured in the framework, and averaged upon finishing. At runtime, the execution of the composed test returns the total elapsed time, and as a side-effect, it produces the partial elapsed times of the involved services for which a performance test module was included in the composition. 


\section{Test Coverage}

The third form of composition supported by PASCANI targets test coverage. As mentioned previously, test coverage is used in classical testing for evaluating the thoroughness of a set of test artifacts, measured as the percentage of code whose execution is run-through by the test suites execution. More concretely, test coverage uses a control flow-based coverage criteria whose goal is to execute every statement in a program, or specified blocks of it. It is worth mentioning that several approaches for automating the generation of test data-sets have been proposed to achieve an exhaustive coverage of all control flow paths (e.g., [12], [13]) even though it is known that this is unfeasible in general. In any case, automated generation of these data sets is outside the scope of this paper, as it addresses another dimensions of the testing problem.

In PASCANI, test coverage is evaluated as the thoroughness of a set of test modules, measured as the percentage of the services that are actually tested, with respect to the total number of provided services in all components subject to test (i.e., imported in the system structure block of the PASCANI test module specification). The test modules are executed in sequence, avoiding repetitions in them and in service execution. The composition of several test modules $T M_{1}, \ldots, T M_{N}$ for measuring their coverage is realized through the definition of the test-coverage operator, denoted as $\odot$, and expressed in the language as coverage $\left\{T M_{1}, \ldots, T M_{N}\right\}$.

Definition 3: Given a set of test modules $T S=$ $\left\{T M_{1}, \ldots, T M_{N}\right\}$, the test coverage of $T S$, denoted as $T M_{1} \odot$ $\ldots \odot T M_{N}$ and abbreviated $\bigodot_{i=1}^{N} T M_{i}$, is defined as:

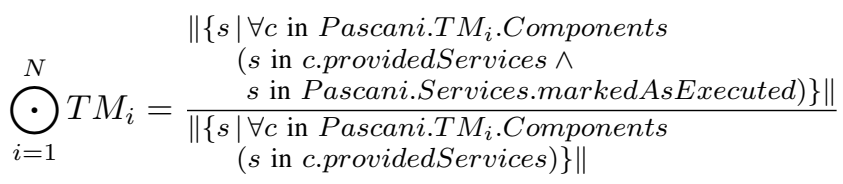

where $\left\{s \mid \forall c\right.$ in Pascani.T $M_{i}$.Components $(s$ in c.provided Services)\} is the defined-by-comprehension set of the provided services $s$ that are defined in all of the components imported in test module $\mathrm{TM}_{i}$; and $\|X\|$ is the cardinality of the set $X$ ( $\left.\frac{\text { count }(\text { testedServices })}{\text { count }(\text { totalServices })} * 100 \%\right)$.

That is, as with the performance-testing composition operator, PASCANI includes a record of the executed services in the attributes associated to every test module $\mathrm{TM}_{i}$, which is updated dynamically at runtime as the services involved in the test modules are executed. In this definition, Pascani. $\mathrm{TM}_{i}$. Components refers to all of the imported components in the system structure block of $\mathrm{TM}_{i}$, whereas Pascani.Services.markedAsExecuted refers to the services actually executed in the given execution.

Thus, the semantics of the test-coverage composition operator not only computes the ratio between the number of actually tested services and the total number of them (expressed as a fraction of $100 \%$ ), but also activates the recording of the executed services in the generated configuration. At runtime, the tests execution returns the percentage of test coverage, and as a side-effect, produces the record of the services that were actually tested (in cascade) from these tests execution. The total number of defined services is obtained through the
PASCANI analyzer. Both functionalities, the update of test module attributes and the count of the total number of defined services in the set of imported components, are supported trough an extension of the FRASCATI SCA middleware and its introspection capabilities developed by Tamura et al. [14].

\section{Evaluation}

This section presents an evaluation of two aspects of PASCANI: its practical feasibility for composing testing services, and the relative gain it offers in development effort for specifying testing artifacts. To conduct this evaluation, we analyze the application of PASCANI in the service provider side using the matrix-chain multiplication (MCM) problem. Even though we also applied PASCANI for the service integrator side in the OSR application, we omit details of the respective results due to space constraints.

\section{A. Composing Tests: the Service Provider Side}

Assume the MCM service provider splits the matrix-chain multiplication problem into three different subproblems: (i) the matrix-pair multiplication problem; (ii) the matrix-chain parenthesization problem, which finds the multiplication order that minimizes the number of scalar multiplications; and (iii) the matrix-subchain multiplication scheduling problem, which takes the output from (ii) and finds subsets of matrix multiplications that can be executed concurrently to decrease the total multiplication time [15]. Fig. 4 depicts the variability of the MCM problem in the form of a feature model. ${ }^{1}$ The solutions for each of these three subproblems are represented as features MatrixpairMultiplicator, Parenthesizer, and Scheduler, respectively (cf. second level in Fig. 4). Moreover, each feature in the model is associated with an SCA component.

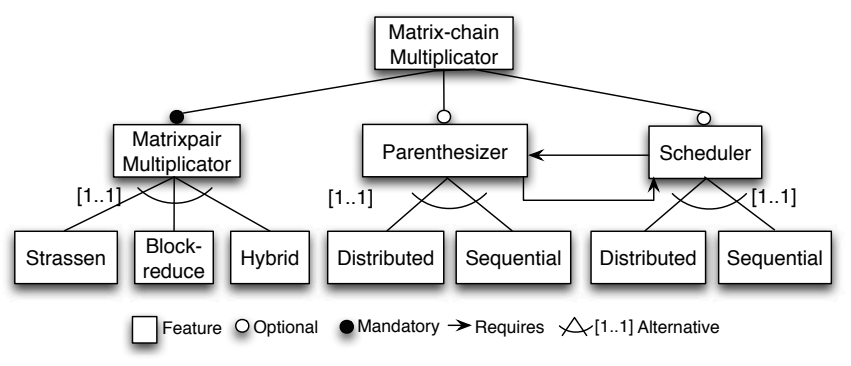

Figure 4: Feature model for the matrix-chain multiplication solution.

To realize the possible configurations of the MCM service, we implemented seven SCA components. For solving the matrix-pair multiplication problem we implemented three alternative components: Strassen, BlockReduce, and Hybrid. Strassen implements the sequential solution proposed by Volker Strassen, BlockReduce implements a map-reduce strategy, and Hybrid implements a variation of the block-reduce strategy. For solving the matrix-chain parenthesization and the matrix-subchain problems, we implemented two alternative components for each case: Sequential and Distributed.

\footnotetext{
${ }^{1}$ This model is available in the SPLOT tool: http://goo.gl/nDhUR5
} 
The fundamental motivation behind PASCANI is the composition and reuse of test modules, which is achieved through the specification of unit, integration, and system test modules. PASCANI allows the specification of integration and system tests by invoking the test cases of imported modules, and the composition of test modules through the regression $(\uplus)$, performance $(\cap)$, and coverage $(\odot)$ operators presented in Section IV. In light of this, each component of the MCM solution is equipped with one or several basic test modules that address different test objectives. We implemented basic test modules for testing the correctness and performance of several of the SCA components used in the MCM problem of our case study. For example, component Strassen is accompanied by a basic test module $T M_{\text {StrassenTest }}$ that tests its correctness. The target of these two modules is unit testing, in its functional and extra-functional aspects, respectively.

In this case study, PASCANI supports the service provider in the implementation and execution of the integration and system tests required to validate the correctness and performance of each of the fifteen MCM products that can be obtained from the variability model presented in Fig. 4. The explanations in this section use two of these possible products: $M C M_{1}$ and $M C M_{2}$. Assume that product $M C M_{1}$ is defined by component MatrixpairMultiplicator. Strassen (also referred as Strassen for the sake of simplicity), whereas product $\mathrm{MCM}_{2}$ by components Strassen, Parenthesizer.Sequential and Scheduler.Sequential. The tester must specify test modules that call the test control blocks or selected test cases of the modules associated with the SCA components that define each product configuration. For example, for testing the correctness of product $\mathrm{MCM}_{2}$, the tester must specify an integration module $T M_{\text {IntegrationTest }}$ whose execution produces a verdict on the integration. This verdict results from the and operation applied to the values returned by the basic test modules in charge of testing the individual correctness of its three components.

1) Regression Testing: Suppose now that the implementation of component Strassen is modified. Because of this change, which does not affect the component service interfaces, the tester must implement a regression test to guarantee that product $M C M_{2}$ still satisfies its functional or extra-functional requirements. For this, the tester must create a composed test module $T M_{\text {RegressionTest }}$ that specifies in its control block a regression test that composes the unit test of component Strassen with the integration test of product $M C M_{2}$ using the regression operator: $T M_{\text {StrassenTest }} \uplus T M_{\text {IntegrationTest. That is, if the }}$ Strassen component implementation is changed (because e.g. of a new version or an evolutionary change of the algorithm base), it is necessary to retest that both the unit tests of the component correctness and its integration test with the Parenthesizer and Scheduler components are still successfully passed. Fig. 5 depicts the SCA (partial) configuration generated by PASCANI for the regression test module $T M_{\text {RegressionTest }}$ according to the regression composition semantics. Section (A) in the figure, above the horizontal dashed line, corresponds to the SCA configuration of the integration module $T M_{\text {IntegrationTest }}$ and the basic module $T M_{\text {StrassenTest. }}$ Component IntegrationTest implements the test strategy specified in the control block of the integration module, whereas component StrassenTest (D) implements the unit test that checks the correctness of component Strassen. Component Regressiontest in section (B) implements the test strategy for the regression test, which involves the execution of the StrassenTest, the unit test, and IntegrationTest, the integration test.

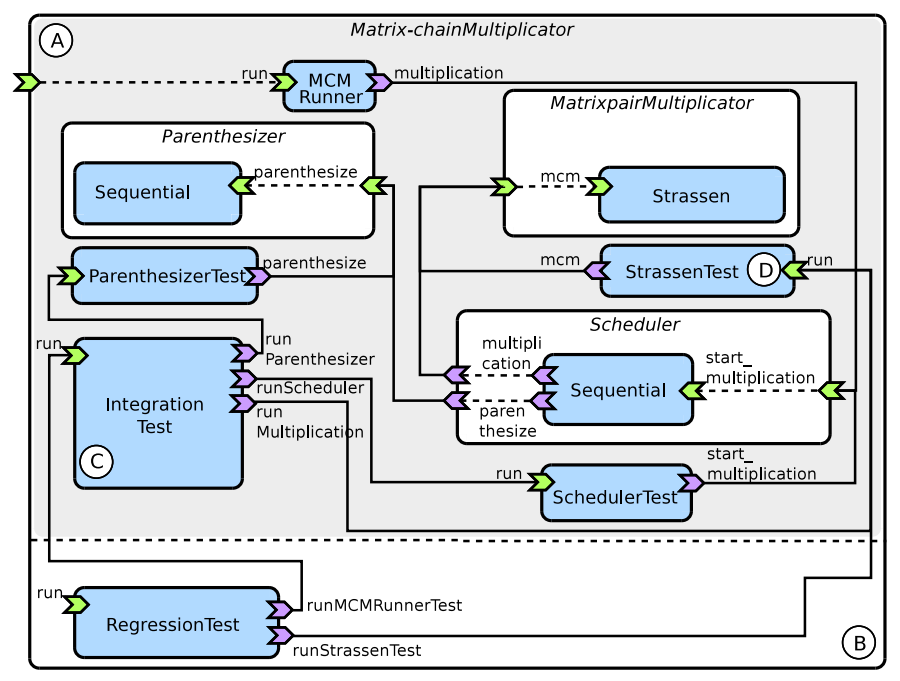

Figure 5: SCA (partial) configuration for the regression test.

A regression test report in PASCANI typically includes, for each test module, its total execution time; a flag to indicate whether the module was executed in regression test mode; and for each of the services tested by its test components, a general flag to indicate whether the service was tested, and the execution time and verdict for each test case associated with the tested service.

2) Performance Testing: To test the performance of product $M C M_{2}$, the tester must compose the primitive modules that test the performance of components Strassen, Parenthesizer.Sequential, and Scheduler. Sequential: TM PStrassen $\mathrm{C} T M_{P P a r S e q}$ ก $T M_{P S \text { chedSeq }} \leq$ Target $_{\text {maxLimit }}$. From this composed module, PASCANI generates the corresponding SCA configuration. The performance reports, not shown in this paper due to space constraints, contains the general verdict and performance measures obtained for product $M C M_{2}$, as well as the verdicts and measures for each individual component. This level of detail in the logging of test results is possible thanks to the composition semantics of the performance tests operator, and the tracing and logging facilities of PASCANI.

Table I shows the outputs obtained when using the performance composition operator to test products $M C M_{1}$ and $M C M_{2}$ for the multiplication of matrices of sizes $640 \times 480$, $480 \times 320,320 \times 240$, and $240 \times 640$ among others.

3) Test Coverage: The composition of coverage tests is specified similarly as the regression and performance tests composition: a module must be created, using the coverage operator $(\odot)$ to compose the test modules for which the measure is required. For example, the coverage achieved when composing the basic test modules associated to $\mathrm{MCM}_{2}$ : T $M_{\text {StrassenTest }} \odot T M_{\text {ParenthesizerTest }} \odot T M_{\text {SchedulerTest }}$ corresponds to $100 \%$, because the composition has three 


\begin{tabular}{lcc}
\hline Component & $M C M_{1}(\mathrm{~ms})$ & $M C M 2(\mathrm{~ms})$ \\
\hline Parenthesizer & - & 1 \\
Scheduler & - & 1 \\
Strassen & 3447 & 4774 \\
Total time & 3449 & 4774 \\
\hline
\end{tabular}

Table I: Performance tests results for products $M C M_{1}$ and $M C M_{2}$.

services to test: Scheduler.start_multiplication, Parenthesizer.parenthesize, and Strassen.mcm, and each service is tested by the corresponding basic test module. However, as the complexity and number of component tests grow, the more difficult is to determine the percent of coverage manually.

\section{B. Engineering Effort}

Table II presents the relative gain of developer's effort when specifying tests with PASCANI in our case study. The results show an effort gain superior to $70 \%$ for all cases. This is mainly because PASCANI releases the developer from implementing functionalities of the SCA layer, such as protocol translation and message marshaling and unmarshaling.

In this table, column Component refers to the service components we tested. For evaluation purposes, each test case was defined in a separate test module (cf. column Test Module) thus leaving the measurement at the coarsest level of granularity. Column PASCANI LOCs refers to the number of lines of code written in PASCANI by the developer. Column Generated LOCs includes two sub-columns: Project, which refers to the classes that are automatically generated in correspondence with the test modules; $X M L$, which refers to the generated SCA composites. Finally, column Effort refers to the developer's effort when writing tests with PASCANI, and column Reduction the relative effort gain obtained when using PASCANI.

\section{RELATED WORK}

This section presents related work in light of the categories of challenges discussed in Section II-A: composability, traceability and logging, test specification and expressiveness.

Concerning composability, we found no approaches that support the composition of testing artifacts for testing software services. Component compositions may have an important number of variation sources, which increase exponentially with the number of individual testing component options. Therefore, the composition of existing unit tests can help reduce the costs of the testing process.

The lack of composable and reusable testing artifacts limits the effectiveness of the validation process in service-oriented systems [7], [4]. This is in part because of the extramural nature of services to compose that makes impractical to anticipate all the possible deployment configurations of the composition, thus test cases cannot be executed in the real context of execution, which involves the context of third parties such as their computational infrastructure or load. Moreover, systems implement adaptive behaviors through dynamic binding, either by replacing individual services or adding new ones, and thus integration testing has to deal with changing configurations that cannot be anticipated [16]. To counteract this issue, several approaches rely on simulation and prediction mechanisms to mimic third party components and evaluate their behavior and properties [17], [18]. PASCANI provides suitable mechanisms not only to instrument services with testing services, but also to specify and implement test cases that can easily compose these testing services at any time according to changes in service compositions.

Regarding traceability and logging, the lack of instrumentation to support these functions in service testing augments the complexity of understanding component behaviors and reporting errors. Even though we found no evidence of research works focused on the traceability of tests at every level, in general logging in service testing is better instrumented for unit tests than in integration or system tests. Nevertheless, keeping track of external behaviours is still an open challenge for distributed environments. PASCANI not only provides logging mechanisms applicable to individual tests and their compositions, but also these mechanisms are automatically deployable since they are generated as part of the test modules. PASCANI also allows software testers to select the types of traces applicable in a particular situation, or decide on the level of logging (e.g., by enabling logging functionalities for a selected set of test cases).

Finally, with respect to test specification and expressiveness, we found only one domain specific language (DSL), based on ontologies [19], for the specification of tests in service-oriented applications. However, we found no DSLs suitable for the specification of reusable, composable, and traceable service-oriented tests. In general, the focus is on generating test components or test logic from service specifications, in particular WSDLs, rather than on the specification of tests themselves [17], [20]. PASCANI defines composition operators whose semantics guarantee that the generated testing components automatically have these characteristics. PASCANI is a suitable and easy to use language intended for developers to specify composable testing artifacts for SOA/SCA applications. In this category, it is worth mentioning that most of the PASCANI language testing concepts were borrowed from TTCN-3 (cf. http://www.tten-3.org). TTCN-3 is a test specification and implementation language designed by the European Telecommunications Standards Institute (ETSI), for the specification of reactive system tests over a variety of communication interfaces. It has been widely used for testing protocols as well as embedded, communication-based, and distributed systems. Despite TTCN-3 is a standardized language and provides a full stack for testing software at any stage, its syntax and semantics remain very close to lowlevel communication testing, therefore extending it for business application developers was not a suitable option. From TTCN3 we adopted and adapted its modular approach, its concept of executable test suites, and the semantics for test case resolution into verdicts.

\section{CONCLUSION}

In this paper we presented PASCANI, a framework for specifying, and executing automated, composable, and traceable tests for service-oriented systems. Test modules in PASCANI are structured in three definition blocks: the system 


\begin{tabular}{|c|c|c|c|c|c|c|}
\hline \multirow{3}{*}{$\begin{array}{l}\text { Component } \\
\text { Strassen }\end{array}$} & \multirow{3}{*}{$\begin{array}{l}\text { Test Module } \\
\text { checkCorrectness } \\
\text { compareWithClassicStrassen }\end{array}$} & \multirow{3}{*}{$\begin{array}{c}\text { PASCANI } \\
\text { LOCs } \\
50 \\
54 \\
\end{array}$} & \multicolumn{2}{|c|}{$\begin{array}{l}\text { Generated LOCs } \\
\text { (Project) (XML) }\end{array}$} & \multirow{3}{*}{$\begin{array}{l}\text { Effort } \\
23.92 \% \\
25.59 \%\end{array}$} & \multirow{3}{*}{$\begin{array}{c}\text { Reduction } \\
76.08 \% \\
74.41 \%\end{array}$} \\
\hline & & & 155 & 54 & & \\
\hline & & & 157 & 54 & & \\
\hline \multirow{2}{*}{ HybridMultiplication } & checkCorrectness & 45 & 143 & 54 & $22.84 \%$ & $76.16 \%$ \\
\hline & checkExecutionTime & 34 & 136 & 54 & $17.89 \%$ & $82.11 \%$ \\
\hline NMatrices & checkExecutionTime & 30 & 127 & 54 & $16.57 \%$ & $83.43 \%$ \\
\hline \multirow[t]{2}{*}{ MatrixChainMultiplication } & compareMethods & 35 & 479 & 181 & $5.30 \%$ & $94.70 \%$ \\
\hline & isServiceAlive & 19 & 126 & 54 & $10.56 \%$ & $89.44 \%$ \\
\hline \multirow[t]{2}{*}{$\mathrm{CCNV}$} & testCreditCardPartners & 50 & 151 & 54 & $24.39 \%$ & $75.61 \%$ \\
\hline & testCreditCardNumbers Verification & 55 & 151 & 54 & $26.83 \%$ & $73.17 \%$ \\
\hline \multirow{3}{*}{ PAV } & isServiceAlive & 24 & 136 & 54 & $12.63 \%$ & $87.37 \%$ \\
\hline & verifyClientAddress & 51 & 134 & 54 & $27.13 \%$ & $72.87 \%$ \\
\hline & verifyZipCodeCities & 27 & 130 & 54 & $14.67 \%$ & $85.33 \%$ \\
\hline
\end{tabular}

Table II: Relative effort gain when specifying tests with PASCANI

structure block, the test cases block, and the test control block. This block structure is a key factor for achieving reusability, composability, and traceability of test specifications. The objective of the system structure block is to define the services and component-based software structure to test, by importing not only system components, but also third-party services and other testing components, being this a mechanism that allows scalability in the definition of tests modules. The test cases and test control blocks allow to effectively reuse and compose testing components generated from test module specifications, through the invocation of imported services and other testing components. This reuse and composition is supported through the exposure and automatic binding of standard services for controlling traceability and test logging, which are generated at compile time. Our results show the applicability of the framework by generating, deploying and executing SCA configurations of composable tests. Moreover, these results evidence the benefits that PASCANI offer, by saving considerable time and effort in the testing development process for human testers.

\section{REFERENCES}

[1] M. Beisiegel, H. Blohm, D. Booz, M. Edwards, O. Hurley et al., "Service Component Architecture, Assembly Model Specification," OSOA, Specification Version 1.0, 2007. [Online]. Available: http://www.osoa org/display/Main/Service+Component+Architecture+Specifications

[2] L. Seinturier, P. Merle, R. Rouvoy, D. Romero, V. Schiavoni, and J.-B. Stefani, "A Component-Based Middleware Platform for Reconfigurable Service-Oriented Architectures," Software: Practice and Experience, vol. 42, no. 5, pp. 559-583, May 2012.

[3] M. J. Harrold, "Testing: A Roadmap," in Procs. of Conf. on the Future of Software Engineering, ser. ICSE 2000. ACM, 2000, pp. 61-72.

[4] G. Canfora and M. D. Penta, "Testing Services and Service-Centric Systems: Challenges and Opportunities," IT Professional, vol. 8, no. 2, pp. 10-17, 2006.

[5] F. Buschmann, "Tests: The Architect's Best Friend," IEEE Software, vol. 28, no. 3, pp. 7-9, 2011.

[6] M. P. Papazoglou, P. Traverso, S. Dustdar, and F. Leymann, "ServiceOriented Computing: State of the Art and Research Challenges," Computer, vol. 40, pp. 38-45, November 2007.

[7] J. Gao, E. Zhu, and S. Shim, "Tracking Software Components," Journal of Object Oriented Programming, 2001.
[8] J. Z. Gao, J. Tsao, Y. Wu, and T. H.-S. Jacob, Testing and Quality Assurance for Component-Based Software. Norwood, MA, USA: Artech House, Inc., 2003.

[9] G. Karsai, H. Krahn, C. Pinkernell, B. Rumpe, M. Schindler, and S. Völkel, "Design Guidelines for Domain Specific Languages," in 9th OOPSLA Workshop on Domain-Specific Modeling, 2009.

[10] Z. Huang, D. Zeng, and H. Chen, "A Comparison of CollaborativeFiltering Recommendation Algorithms for E-commerce," Intelligent Systems, IEEE, vol. 22, no. 5, pp. 68-78, Sept 2007.

[11] A. Abran, P. Bourque, R. Dupuis, J. W. Moore, and L. L. Tripp, Eds., Guide to the Software Engineering Body of Knowledge - SWEBOK. Piscataway, NJ, USA: IEEE Press, 2004.

[12] Z. Wang, S. Elbaum, and D. S. Rosenblum, "Automated Generation of Context-Aware Tests," in Procs. of 29th Intl. Conf. on Software Engineering, ser. ICSE 2007. Washington, DC, USA: IEEE Computer Society, 2007, pp. 406-415.

[13] K. Kähkönen, "Automated Test Generation for Software Components," Helsinki University of Technology, TKK Reports in Information and Computer Science, Tech. Rep., 2009.

[14] G. Tamura, R. Casallas, A. Cleve, and L. Duchien, "QoS Contract Preservation through Dynamic Reconfiguration: A Formal Semantics Approach," Science of Computer Programming (SCP), pp. 1-30, 2014.

[15] H. Lee, J. Kim, S. J. Hong, and S. Lee, "Processor Allocation and Task Scheduling of Matrix Chain Products on Parallel Systems," IEEE Transactions Parallel and Distributed Systems, vol. 14, no. 4, pp. 394407, 2003.

[16] G. Canfora and M. Penta, "Service-oriented architectures testing: A survey," in Software Engineering, A. Lucia and F. Ferrucci, Eds. Berlin, Heidelberg: Springer-Verlag, 2009, pp. 78-105.

[17] J. Yu, J. Han, J.-G. Schneider, C. Hine, and S. Versteeg, "A Virtual Deployment Testing Environment for Enterprise Software Systems," in Procs. of 8th Intl. Conf. on Quality of Software Architectures, ser. QoSA '12. New York, NY, USA: ACM, 2012, pp. 101-110.

[18] M. Palacios, J. Garca-Fanjul, and J. Tuya, "Testing in Service Oriented Architectures with dynamic binding: A mapping study," Information \& Software Technology, vol. 53, no. 3, pp. 171-189, 2011.

[19] X. Bai, S. Lee, W.-T. Tsai, and Y. Chen, "Ontology-based test modeling and partition testing of web services," in Web Services, 2008. ICWS 2008. IEEE International Conference on, Sept 2008, pp. 465-472.

[20] A. Chaturvedi and A. Gupta, "A tool supported approach to perform efficient regression testing of web services," in In Procs. 7th Intl. Symp. on the Maintenance and Evolution of Service-Oriented and Cloud-

Based Systems (MESOCA), Sept 2013, pp. 50-55. 